\title{
Molecular basis of essential fructosuria: molecular cloning and mutational analysis of human ketohexokinase (fructokinase)
}

\author{
David T.Bonthron*, Nlcola Brady, lain A.Donaldson' and Beat Steinmann² \\ Human Genetics Unt, Unversity of Edinburgh, Western General Hospital, Edinburgh EH4 2XU, 1Department of Biochemistry, University of Oxford, \\ South Parks Road, Oxford OX1 3QU, UK and 2Division of Metabolism, Department of Paediatrics, Steinwiesstrasse 75, CH-8032 Zürich, Switzerland
}

Essentlal fructosuria is one of the oldest known inborn errors of metabolism. It is a benign condition which is believed to result from deficlency of hepatic fructokInase (ketohexokinase, KHK, E.C.2.7.1.3). This enzyme catalyses the first step of metabolism of dietary fructose, conversion of fructose to fructose1-phosphate. Desplte the early recognition of this disorder, the primary structure of human KHK and the molecular basis of essential fructosurla have not been prevlously defined. In thls report, the isolation and sequencing of full-length cDNA clones encoding human ketohexokinase are described. Alternatlve mRNA species and alternative KHK isozymes are produced by alternatlve polyadenylation and splicing of the $K H K$ gene. The KHK proteins show a high level of sequence conservation relatlve to rat KHK. Dlrect evidence that mutation of the $K H K$ structural gene is the cause of essentlal fructosurla was also obtained. In a wellcharacterized famlly, in which three of elght siblings have fructosuria, all affected individuals are compound heterozygotes for two mutations Gly40Arg and Ala43Thr. Both mutations result from $G \rightarrow A$ transitions, and each alters the same conserved region of the KHK protein. Neither mutation was seen in a sample of $\mathbf{5 2}$ unrelated control individuals. An additional conservative amino acid change (Val49lle) was present on the KHK allele bearing Ala43Thr.

\section{INTRODUCTION}

In mammals, dietary fructose is primarily metabolized through a pathway distinct from that responsible for glucose metabolism. This pathway utilizes three specialized enzymes, fructokinase (ketohexokinase, KHK, EC 2.7.1.3), aldolase B (fructose-1phosphate aldolase, EC 4.1.2.13) and triokinase (EC 2.7.1.28), which convert fructose into intermediates of the glycolytic and gluconeogenic pathways. KHK is found predominantly in liver, kidney, and small intestine, and catalyses the conversion of fructose to fructose-1-phosphate. It is also active using other ketose sugars as substrate (for review, see ref. 1).

Human KHK has been purified from liver, to a specific activity which suggests it constitutes about $0.07 \%$ of liver protein (2).
Both the human and bovine enzymes appear to be dimers $(2,3)$, with an estimated subunit molecular weight of 39000 . Human KHK has an apparent $K_{m}$ for fructose of $0.86 \mathrm{mM}(2)$; its high $\mathrm{V}_{\max }$ allows very rapid metabolism of dietary fructose via the specialized fructose pathway, which bypasses the usual major site of glycolytic regulation (phosphofructokinase). After parenteral fructose administration, this can result in severe lactic acidosis even in normal individuals (for review, see ref. 1).

Essential fructosuria (MMM number 229800), although not one of the four disorders discussed by Garrod, can claim to be one of the earliest described inborn errors of metabolism, having been first recognized almost 120 years ago. It is a benign condition characterized by the intermittent appearance of fructose in the urine. In affected subjects, ingestion of dietary fructose, sucrose or sorbitol is followed by an abnormally large and persistent rise in blood fructose concentration, and by excretion of 10-20\% of the ingested load in the urine (1). Essential fructosuria appears to be inherited as an autosomal recessive trait (see Discussion). In one affected individual an indirect enzyme assay was used to demonstrate a deficiency of hepatic fructokinase (4), but the molecular basis for essential fructosuria has remained otherwise undefined.

No molecular genetic studies of human $K H K$ have been previously undertaken. The primary structure of rat liver KHK was, however, recently described (5). This work demonstrated that KHK is structurally unlike other mammalian hexokinases, and does not show significant homology with other known mammalian protein families. However, short sequence motifs shared with some bacterial phosphotransferases, which like KHK have a furanose sugar substrate, did suggest the possibility of an ancestral furanose kinase, from which these proteins evolved.

\section{RESULTS}

Isolation of human $K H K$ cDNA clones

Since no other information was available, as to which residues of KHK might be conserved between mammals, we chose to screen for human $K H K$ by low-stringency hybridization with the entire rat $K H K$ coding region, rather than by polymerase chain reaction (PCR) with degenerate oligonucleotide pools. Three cDNA clones $>1 \mathrm{~kb}$ in size, isolated in this way from a cDNA library of the hepatoblastoma cell HepG2, were completely sequenced. The nucleotide sequence and translated open reading frame of the longest of these (pHKHK3a) are shown in Figure

*To whom correspondence should be addressed 


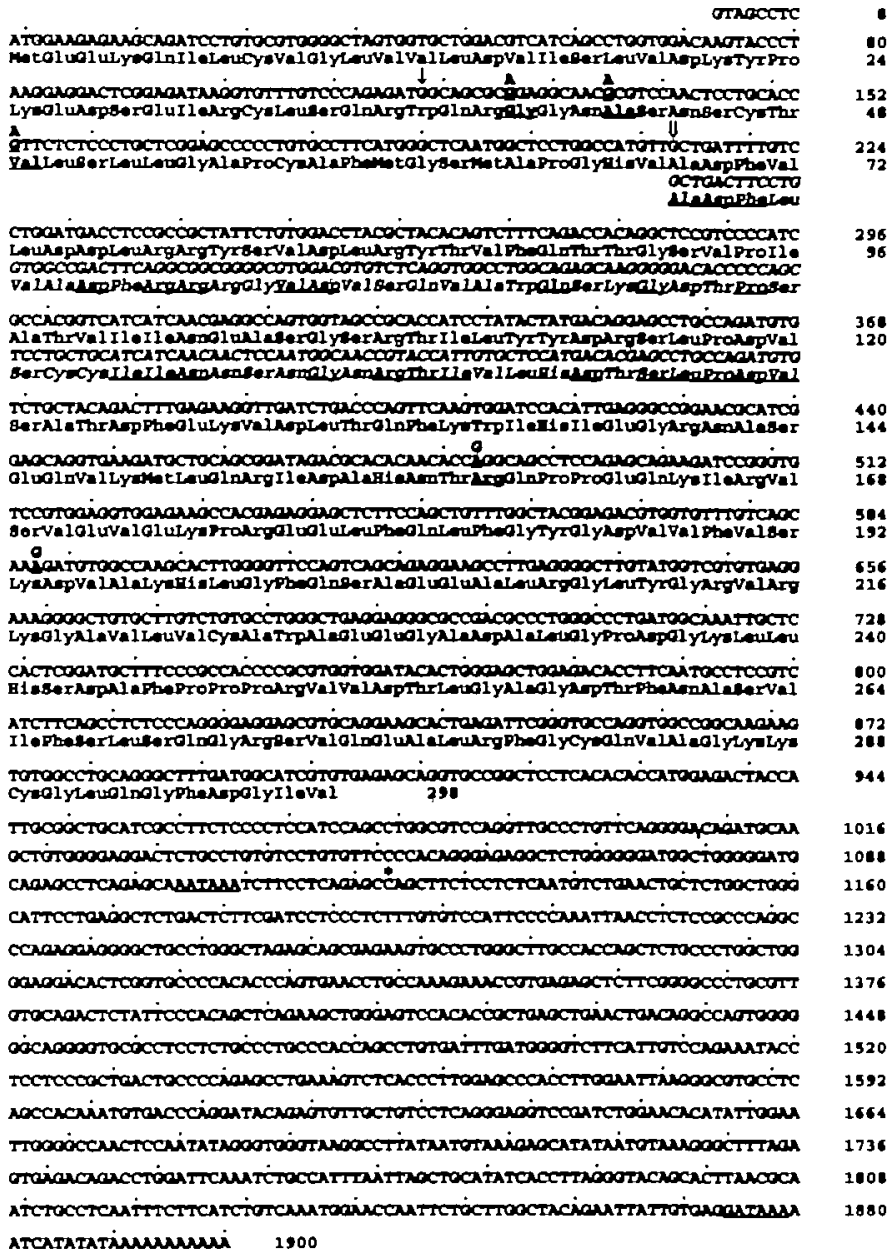

Figure 1. The sequence of $K H K$ cDNA. The sequence of the longest clone (pHKHK3a) is shown. The shorter clones pHKHK3d and pHKHK1-2 lie within this sequence; their 5' limits are indicated by $\downarrow$ and $\square$, respectively. Both of these shorter clones end at the upstream polyadenylation site indicated by *. The consensus and variant polyadenylation signals are underlined. The altematively spliced exon (C) encoded in clone pHKHK1-2 is shown in italics below the main sequence. This exon corresponds closely to the sequence of the rat $K H K \mathrm{cDNA}$ (5; see also Figure 2). Matching residues between the two altermative splice forms of human KHK are underlined. The precise limits of the alternatively spliced exon cannot be stated at present since genomic clones have yet to be analysed. The Gly40Arg and Ala43Thr changes are caused by the mutations indicated in botd type at nt 126 and 143. The other sequence variants observed are also indicated in the same way. Arg 159Gly was found only in clone pHKHK3d, and it is unclear at present whether it represents a cloning artefact or a true polymorphic variant. The nucleotide sequence data reported in this paper will appear in the EMBL, GenBank and DDBJ Nucleotide Sequence Databases under the accession numbers $X 78677$ and $X 78678$.

1. The positions of the sequence variants detected in other clones (discussed below) are also shown.

\section{Alternative splicing and polyadenylation}

The most notable feature of pHKHK3a is its long (984 nt) 3' untranslated region. This is in contrast to the $186 \mathrm{nt} 3^{\prime}$ untranslated region of the published rat $K H K \mathrm{mRNA}$. The other two human clones (pHKHK3d, pHKHK1-2), however, are derived from mRNA which has utilized an upstream polyadenylation site, closer to the position of that in the rat (giving a $3^{\prime}$ untranslated region of $218 \mathrm{nt}$ ).
The sequences of pHKHK3a, pHKHK3d and pHKHK 1-2 are collinear except for nt 218 to 352 in Figure 1. Here, pHKHK1-2 differs over its first $140 \mathrm{bp}$ from the sequence of the other two clones. This appears to result from alternative splicing to include either of two exons. The published rat cDNA sequence (5) corresponds in this region to the splice variant found in pHKHK 1-2, referred to subsequently as form C. The other splice variant is referred to below as form A. Closer inspection (Figure 1) shows that these two alternatively spliced exons can be aligned with each other to reveal conservation of several amino acid residues. This suggests that an intragenic duplication event may have given rise to the alternative exons in this region of the $K H K$ gene.

The predicted molecular weight of the human KHK A subunit is 32 734. The initiator ATG shown in Figure 1 is presumptive, since no in-frame upstream stop codon is present in the clones. However, this assignment is based on the position of the initiator codon in the rat mRNA (5) which was verified by the agreement of the predicted protein $\mathrm{M}_{\mathrm{r}}$ with the $\mathrm{M}_{\mathrm{r}}$ directly determined by mass spectrometry.

A single amino acid variant was found among the three independent HepG2 $K H K$ cDNA clones. Arg159 is altered to Gly by an $A \rightarrow G$ transition in clone pHKHK $3 d$. The alignment in Figure 2 shows that unlike the two residues mutated in essential fructosuria patients (see below) Arg159 is not conserved between human and rat, and indeed lies at the centre of the most poorly conserved region of KHK.

\section{Tissue distribution of $K H K$ mRNA}

KHK activity is found principally in liver, kidney, and small intestine (2); pancreatic islet cells also possess a similar activity (6). Because of this tissue specificity, it was initially unclear whether analysis of $K H K$ RNA in patients with essential fructosuria would be possible. To investigate this, reverse transcription and PCR (RT-PCR) of the $5^{\prime}$ coding region (nt $1-410$, primers KHK $1 / \mathrm{KHK} 3$ ) were performed on RNA from human fetal tissues. As expected, strong signals were obtained from liver, kidney, gut and pancreas, as well as (unexpectedly) from spleen. However, we were encouraged to find lower yields of PCR product also from adrenal, muscle, brain and eye, as well as from human diploid fibroblasts and from an EBVtransformed lymphoblastoid cell line (data not shown). This may be in keeping with previous observations of low level KHK activity in some other tissues (2).

\section{Mutations causing essential fructosuria}

Next, we investigated the postulate that mutation of the human $K K$ gene underlies the rare metabolic disorder, essential fructosuria. The family analysed has been the subject of previous metabolic studies which have demonstrated excessive fructosaemia and fructosuria after oral or intravenous fructose, sorbitol, or sucrose (7-9). After an intravenous fructose bolus (200 $\mathrm{mg} / \mathrm{kg}$ ) the liver concentrations of fructose-1-phosphate, ATP, and phosphate, as determined by ${ }^{31} \mathrm{P}$ magnetic resonance spectroscopy in one affected family member ( $R K$ ), remained unchanged, in contrast to controls, confirming that fructokinase was indeed inactive $(10,11)$. In this Swiss family, three of eight siblings have fructosuria. Since the parents are third degree cousins, it was anticipated that homozygosity for a single mutation would underlie the fructosuria. As the intron-exon structure of $K H K$ remains to be defined, analysis of genomic DNA is difficult. 

Human A
$\Downarrow \Downarrow$
$\Downarrow$.
Rat
Human $c$
WASCTVLSLLGAPCAFMGSMAPGHVADFVLDDLRRYSVDLRYTVFOTTGSVPIATVI 100

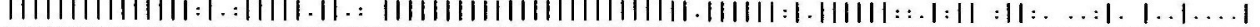
1 MEEKQILCVGLVVDIINVVDYPEEDTDRRCLSQRWQRGGNSNSCTVLSLLGARCAFMGSLAHGHVADFLVADFRRRGVDSQVAWQSQGDTPCSCCI IOO

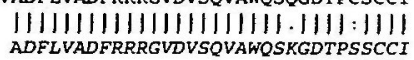
\.
HUMA A 101 INEASGSRTILYYDRSLPDVSATDFEKVDLTQFKWIHIEGRNASEQVKMLORIDAHNTRQPPEQKIRVSVEVEKPREELFQLFGYGDVVFVSKDVAKHLG 200

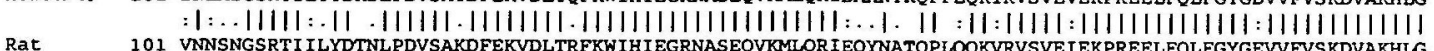 101 VNNSNGSRTI ILYDTNLPDVSAKDFEKVDLTRFKWIHIEGRNASEQVKMLOR IEQYNATQPLQQKVRVSVEIEKPREELFQLFGYGEVVFVSKDVAKHLG 200

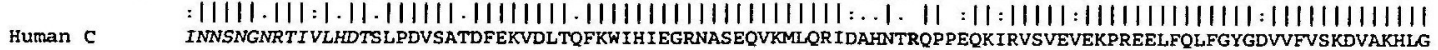
Human 201 FQSAEEALRGLYGRVRKGAVLVCAWAEEGADALGPDGKLLHSDAFPPPRVVTLGAGDTFNASVIFSLSQGRSVQEALRFGCQVAGKKCGLQGFDGIV 298 Rat 201 FRSAGEALKGLYSRVKKGATLICAWAEEGADALGPDGQLEHSDAFPPPRVVDTLGAGDTFNASVIFSLSKGNSMQEALRFGCQVAGKKGLQGFDGIV

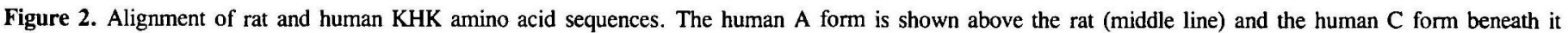

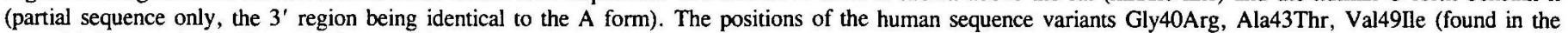

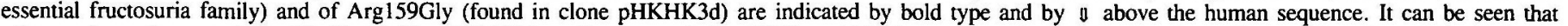

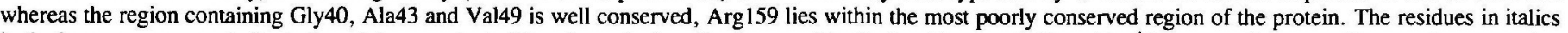

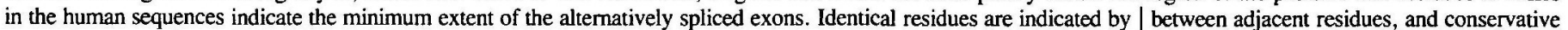
or semi-conservative substitutions by : and ., respectively.

However, the finding of $K H K$ mRNA in lymphoblastoid cells suggested the feasibility of analysing RNA. Therefore, RT - PCR of RNA from a lymphoblastoid cell line from one patient (AK) was performed, amplifying the coding region in two overlapping segments. The PCR products were cloned into the pCRScriptSK + plasmid vector (Stratagene, Inc.) and single overlapping clones were sequenced. The sequence of the entire coding region thus obtained revealed two single-base substitutions. The first, $A \rightarrow G$ at nt 587 , is a silent change at the third base of Lys193. The other, $G \rightarrow A$ at nt 126 , changes the Gly40 codon to Arg. This mutation has occurred at a CpG dinucleotide, within a well-conserved region (Figure 2); it destroys a BstUI site (CGCG) at nt 123-126.

A PCR assay on genomic DNA was then used to analyse for the nt $126 \mathrm{G} \rightarrow$ A change in a sample of 52 normal individuals. None contained the putative mutation, which is therefore unlikely to be a normal polymorphic variant. Next, the same assay was used to type all 10 members of the fructosuria family, by $B s t \mathrm{UI}$ digestion of the 94 bp PCR product (Figure 3a). Surprisingly, this showed that the three fructosuric individuals were heterozygous for Gly40Arg, inherited from their mother. The initial supposition of homozygosity by descent for a single mutation in this consanguineous family was therefore erroneous. Fortuitously though, the other BstUI site predicted in the PCR product (at nt 134-137) was found to be absent from one allele in the father in this family (Figure 3a, lane 1). All three fructosuric patients had also inherited this second sequence variant (Figure 3a, lanes 6, 8, 9). Since the Bst UI site at $134-137$ is also part of an MluI site (ACGCGT), this second mutation was more clearly demonstrated by $M l u I$ digestion of the PCR product (Figure $3 \mathrm{~b}$ ). To define the exact nature of the second mutation, the 94 bp product was digested with MluI and the MluI-resistant $94 \mathrm{bp}$ fraction cloned and sequenced. This revealed two further $\mathrm{G} \rightarrow \mathrm{A}$ changes, at $\mathrm{nt} 135$ and 153 . The first of these causes the non-conservative substitution Ala43Thr and the second the conservative change Val49Ile. Like Gly40Arg, the Ala43Thr mutation was not observed in any of the 52 control DNA samples analysed by $B s t \mathrm{UI}$ digestion, and hence is unlikely to be a polymorphic variant. Separate analysis of the Val49Ile substitution showed the father in this family to be homozygous for Ile 49 and

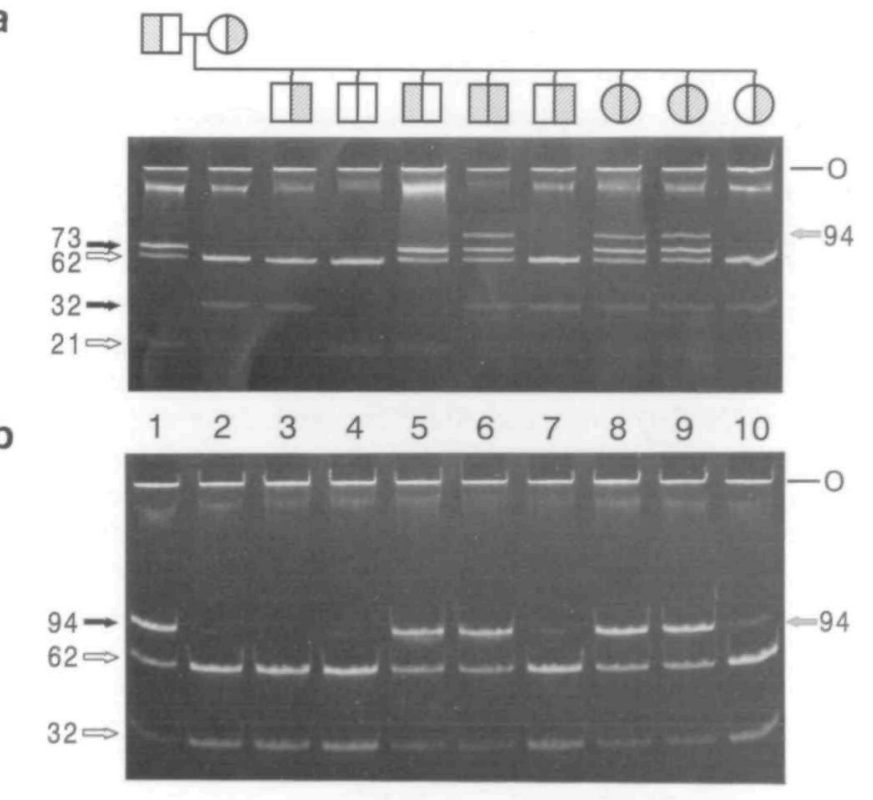

Figure 3. DNA analysis of a family with essential fructosuria. On the pedigree, $K H K$ mutations are indicated by shading of the right (Gly40Arg) or left (Ala43Thr) half of the symbol. (a) $21 \%$ polyacrylamide minigel showing ethidium bromidestained BstUI digests of a 94 bp PCR product (KHK14 - KHK15) containing the Gly40Arg and Ala43Thr mutation sites. The grey arrow to the right indicates the position of undigested product (not present on this gel) and of the uncut heteroduplex bands in the affected individuals in lanes 6,8 and 9 . To the left, white arrows indicate the normal digestion products $(62,21$ and 11 (not seen) bp) and black arrows the fragments of increased size resulting from loss of one or other BstUI site ( $32 \mathrm{bp}$ for Gly $40 \mathrm{Arg}, 73 \mathrm{bp}$ for Ala43Thr). (b) Fifteen percent polyacrylamide minigel showing ethidium bromide-stained MluI digests of the same PCR products. The normal digestion products (white arrows) are 62 and $32 \mathrm{bp}$, the Ala43Thr mutation prevents cleavage ( $94 \mathrm{bp}$, black arrow). The faint $94 \mathrm{bp}$ bands in lanes 7 and 10 are due to incomplete MluI digestion, which is difficult to overcome with this small fragment. $\mathrm{O}=$ origin.

the mother homozygous for Val49 (not shown). Thus a total of four $K H K$ haplotypes can be defined within the family; Gly 40 Ala43 Ile49 (normal paternal haplotype), Gly40 Ala43 Val49 (normal maternal haplotype), Gly40 Thr43 Ile49 (mutant paternal 
haplotype) and Arg40 Ala43 Val49 (mutant maternal haplotype). All six possible combinations of the four haplotypes are seen in the family, but only Gly40 Thr43 De49/Arg40 Ala43 Val49 results in fructosuria.

\section{DISCUSSION}

In this report, we demonstrate that $K H K$ structural gene defects underlie essential fructosuria. The family analysed has been the subject of previous metabolic studies $(7-11)$. However, because of the tissue specificity of $K H K$ expression, deficiency of hepatic KHK appears only to have been proven in one fructosuric individual, by enzyme assay on a liver biopsy (4). Essential fructosuria is certainly rare. Lasker estimated its incidence at less than 1 in 130000 , but plausibly suggested this was an overestimate (12). In four of her five families there was parental consanguinity, and the proportion of such families was used to suggest a true frequency nearer one in a million, as well as to support autosomal recessive inheritance. We were surprised, in light of this, to find that in the family we report here the parental consanguinity appears to be coincidental, with the affected individuals being compound heterozygotes for the mutations Gly40Arg and Ala43Thr.

Since two amino acid substitutions were found together on the paternal $K H K$ allele in our family, we cannot be completely confident that Ala43Thr alone (in the absence of Val49De) is a null mutation. To prove this would require introduction of each change separately into the normal $K H K$ sequence by in vitro mutagenesis and expression. Furthermore, to prove absolutely that Gly40Arg or Ala43Thr are null mutations would require the expression of each mutation in the context of each of the two (A and C) splice variants. None the less, several lines of evidence suggest that Gly40Arg and Ala43Thr are indeed responsible for the fructosuric phenotype. They lie in a conserved region of the protein, produce non-conservative amino acid changes, and are not present in $>100$ control alleles. Val49Me, on the other hand, does not produce fructosuria when present in the homozygous state, and is a common polymorphism in normal Europeans (data not shown). Finally, under the rather unlikely null hypothesis that essential fructosuria could result from a mutation unlinked to $K H K$, the concordant segregation between the mutations and the fructosuria in this family has a probability of only $(1 / 4)^{2}(* / 4)^{5}$ (excluding one proband) $\approx 1$ in 67 .

The most important new finding from these studies of human $K H K$ is that of alternative splicing, due to inclusion of one or other of two similar exons, which we presume arose by an intragenic duplication, though clarification of this must await analysis of genomic $K H K$ clones. It is not known at present whether both splice forms of human KHK are enzymatically active. However, the rat cDNA, which is known to be active when expressed in isolation (13), is close in sequence to the human $C$ form, suggesting strongly that the latter at least will encode a functional enzyme. Since both the human and bovine KHK appear to be dimers in their native state, at least three different KHK isozymes (A-A, A-C and C-C) could exist in viwo. Both mutations we have identified lie in a conserved region, common to both KHK splice forms, so that each could potentially ablate function of all KHK isozymes.

KHK is believed to be synthesized largely in liver, renal cortex, and small intestine, but one previous report also demonstrated KHK-like activity in pancreas (6). Our preliminary RT-PCR experiments are not quantitative but tend to confirm these observations at the RNA level. The distribution of different KHK splice forms in individual tissues requires analysis by RNase protection, and is currently under study. However, preliminary RT - PCR data suggest that the C-type mRNA may be confined to those tissues expressing $K H K$ at high level (liver, kidney, gut, pancreas). It is interesting to note that the gene for glucokinase, another hexokinase displaying a restricted tissue distribution which includes liver and pancreatic islet cell, is subject to celltype specific alternative splicing in both humans and rat $(14,15)$. Characterization of the details of $K H K$ alternative splicing and tissue-specific promoter function await the isolation of $K H K$ genomic clones.

It is uncertain whether the rat $K H K$ gene is also alternatively spliced. However, the considerable divergence between human $\mathrm{A}$ and $\mathrm{C}$ exons, compared with the close similarity between rat and human $C$ exons, suggests that the duplication which produced the alternative exons is ancient, and pre-dated the divergence of rodent and primate lineages.

\section{MATERIALS AND METHODS}

\section{Isolation of human $K H K$ cDNA clones}

The rat liver $K H K$ cDNA construct pUC-KHK-G7 (5) was digested with $B g I I I$ to excise a $0.9 \mathrm{~kb}$ coding region fragment, which was labelled by random priming and used to screen a library (constructed by Dr D.Simmonds, Oxford University) of HepG2 (hepatoblastoma cell line) CDNA in the vector PCDM8 (16), propagated on the supF-selecting strain MC1061/P3. Final washing conditions for the crossspecies hybridization were: $6 \times$ SSC, $0.1 \%$ SDS. Screening of 50000 colonies yielded 13 positive clones, of which three (pHKHK3a, pHKHK3d and pHKHK1-2; insert sizes $2.0,1.15$ and $1.1 \mathrm{~kb}$ ) were completely sequenced directly on doublestranded templates, using synthetic internal or flanking primers.

\section{Cell culture and RT-PCR}

Lymphoblastoid cells were grown in RPMI1640 medium, with 10\% fetal calf serum, $2 \mathrm{mM} \mathrm{L}$-glutamine, and $25 \mu \mathrm{g} / \mathrm{ml}$ gentamicin. Total cell RNA was prepared by the AGPC method (17). Reverse-transcription of $1 \mu \mathrm{g}$ total RNA was performed for $1 \mathrm{~h}$ at $37^{\circ} \mathrm{C}$ in a $30 \mu \mathrm{l}$ reaction mixture containing random hexadeoxynurcleotide primers, $10 \mathrm{mM}$ TrisHCl, pH $8.3,50 \mathrm{mM} \mathrm{KCl}, 6.5 \mathrm{mM} \mathrm{MgCl}_{2}, 10 \mathrm{mM}$ dithiothreitol, $1 \mathrm{mM}$ each $\mathrm{dNTP}, 15 \mathrm{U}$ MuLV reverse transcriptase (BRL-Gibco). For PCR, $15 \mu$ of cDNA synthesis reaction mixure was added directly to $35 \mu \mathrm{l}$ RT - PCR mix (10 mM TrisHCl pH 8.3, $50 \mathrm{mM} \mathrm{KCl}, 1.5 \mathrm{mM} \mathrm{MgCl} 2,285$ nM each PCR primer, $1 \mathrm{mM}$ EDTA, $2 U$ recombinant $T a q$ polymerase). The $K H K$ coding region was amplified in two overlapping segments using primer pairs $\mathrm{KHK} 1 / \mathrm{KHK} 3$ and $\mathrm{KHK} 2 / \mathrm{KHK} 11$. In each case, 40 cycles of $1,1,2 \mathrm{~min}$ at 94 , $63,72^{\circ} \mathrm{C}$ were performed in a Perkin-Elmer Cetus Thermal Cycler. Products were gel-purified prior to cloning.

\section{Genomic DNA analysts}

Genomic DNA was isolated from frozen whole EDTA-blood by Triton X-100 lysis and proteinase $\mathrm{K}$ digestion (18). For typing of the Gly40Arg and Ala43Thr mutations, primers KHK14 and KHK15 were used for 30 cycles of PCR under conditions as above except for $a 60^{\circ} \mathrm{C}$ annealing temperature. The 94 bp product is cleaved by BstUl (CGCG) to $21+11+62$ bp (Gly40 Ala43), to $32+62$ bp (Arg40 Ala43) or to $21+73$ bp (Gly40 Thr43). The small size of the PCR product was dictrted by the apparen positions of splice junctions and the unknown genomic sequence flanking the exons, and by the proximity of the two BstUI sites. The BstUl digestion products were analysed on $21 \%$ polyacrylamide gels.

\section{OAigonucleotide sequences}

KHK1: GTAGCCTCATGGAAGAGAAGC; KHK2: GTGTCTGCTACAGACTTTGAG; KHK3: CTTGAACTGGGTCAGATCAAC; KHK11: AGCTTGCATCTGTCCCCTGAA; KHK14: TTTGTCCCAGAGATGGCAGCG; KHK15: CATTGAGCCCATGAAGGC.

\section{ACKNOWLEDGEMENTS}

We are very grateful to Dr Roderick Campbell for providing samples of human fetal RNA and cDNA, to Dr Gerhard Meng (Würzburg) for establishing lymphoblastoid cell lines from the fructosuric family, to Dr David Simmonds for the HepG2 cDNA library, and to Annete Gilfillan for oligonucleotide synthesis. 


\section{ABBREVIATIONS}

$\mathrm{KHK}$, ketohexolinase; $\mathrm{K}_{\mathrm{m}}$, Michaelis constant; MIM, Mendelian Inheritance in Man; $\mathrm{M}_{r}$, relative molecular mass; PCR, polymerase chain reaction; SDS, sodium dodecyl sulphate; $\mathrm{SSC}$, salt/sodium citrate $(0.15 \mathrm{M} \mathrm{NaCl}, 15 \mathrm{mM}$ sodium citrate, $\mathrm{pH} 7.0$ ).

\section{REFERENCES}

1. Gizzelmann,R., Steinmann, B. and van den Berghe,G. (1989) Disonders of fructose metabolism. In Scriver,C., Beaudet,A. and Valle,D. (ods.), The metabalic basis of inherised disease, 6ih edn. McGraw-Hill, New York, pp. $399-423$.

2. Bais,R., James,H.M., Rofe,A.M. and Conyers,R.A.J. (1985) The purification and properties of human liver ketohexokinase. Biachem. J. 230, 53-60.

3. Raushel,F.M. and Cleland,W.W. (1977) Bovine liver fructokinase: purification and kinetic properties. Biochemistry 16, 2169-2175.

4. Schapira,F., Schapira,G. and Dreyfus,J.-C. $(1961-62)$ La Itsion enzymatique de la fructosurie bénigne. Enzymol. Biol. Clin. 1, 170-175.

5. Donaldson,I.A., Doyle,T.C. and Matas,N. (1993) Expression of rat liver ketohexolinase in yeast results in fructose intolerance. Biochem. J. 291, $179-186$.

6. Malaisse,W.J., Malaisse-Lagae,F., Davies,D.R. and Van Schaftingen,E. (1989) Presence of fructokinase in pancreatic islets. FEBS Lett. 255, $175-178$.

7. Petersen,A., Steinmann,B. and Gitzelmann,R. (1992) Essential fructosuria: increased levels of fructose-3-phosphate in erythrocytes. Enzyme 46, $319-323$.

8. Steinmann,B., Baerlocher,K. and Gitzelmann,R. (1975) Hereditäre Stönungen des Fruktosestoffwechsels: Belastungsproben mit Fruktose, Sorbitol, und Dihydroxyaceton. Nutr. Metab. 18 (Suppl. 1), 115-132.

9. Steinmann,B. and Gitzelmann,R. (1981) The diagnosis of hereditary fructose intolerance. Helv. Paediatr. Acta 36, 297-316.

10. Boesiger,P., Buchli,R., Meier,D., Steinmann,B. and Gitzelmann,R. (1994) Changes of liver metabolite concentrations in adults with disorders of fructose metabolism after intravenous fructose by ${ }^{31} \mathrm{P}$ MRS. Pectiatr. Res. (in press).

11. Boesiger,P., Buchli,R., Meier,D., Steinmann,B. and Gitzelmann, R. (1991) Metabolic changes in liver after intravenous fructose in adults with disorders of fructose metabolism and in healthy controls, followed by phosphorus magnetic resonance spectroscopy. Pediatr. Res. 30, 651 (abstract).

12. Lasker,M. (1941) Essential fructosuria. Hum. Biol. 13, 51-63.

13. Doyle,T.C., Spicket,,C.M., Donaldson,I.A. and Ratcliff,R.G. (1993) Metabolic studies of a fructose-intolerant yeast by in viwo ${ }^{31} \mathrm{P}$-nuclear magnetic resonance spectroscopy. Yeast 9, 867-873.

14. Tanizawa, Y., Koranyi,L.I., Welling,C.M. and Permutt,M.A. (1991) Human liver glucokinase gene: cloning and sequence determination of two alternatively spliced cDNAs. Proc. Nall Acad. Sci. USA 88, 7294-7297.

15. Hayzer,D.J. and Iynedjian,P.B. (1990) Altemative splicing of glucokinase mRNA in rat liver. Biochem. J. 270, 261-263.

16. Seed,B. (1987) An LFA-3 cDNA encodes a phospholipid-linked membrane protein homologous to its receptor CD2. Nature 329, 840-842.

17. Chomezynski,P. and Sacchi,N. (1987) Single-step method of RNA isolation by acid guanidinium thiocyanate-phenol-chloroform extraction. Anal. Biochem. 162, 156-159.

18. Towner,P. (1991) In Brown, T.A. (ed.), Essential Molecular Biology-A Practical Approach. IRL Press, Oxford, pp. 50-53. 
